\title{
Study of quantum current enhancement, eigenenergy spectra and magnetic moments in a multiply connected system at equilibrium
}

\author{
Colin Benjamin 7 and A. M. Jayannavar用 \\ Institute of Physics, Sachivalaya Marg, Bhubaneswar 751 005, Orissa, India
}

(Dated: November 15, 2018)

\begin{abstract}
A multiply connected system in both its open and closed form variations but in equilibrium is studied using quantum waveguide theory. The system exhibits remarkable features, in its open form variation we see current enhancement, hitherto seen only in non-equilibrium systems in absence of magnetic flux. In its closed form analog parity effects are broken. Further we analyse the global and local current densities of our system and also show that the orbital magnetic response of the system calculated from the current densities (and inherently linked to the topological configuration) is qualitatively not same as that calculated from the eigenenergy spectra.
\end{abstract}

PACS numbers: 73.23.-b, 05.60.Gg, 72.10.Bg, 72.25.-b

Mesoscopic systems are those whose dimensions lie between the macroscopic and the atomic scale. Typically the system sizes are in the nano-range. Thus, mesoscopic physics addresses fundamental problems which occur when a macroscopic object is miniaturized. Over the past decade, research into the electrical transport properties of mesoscopic systems has provided insight into fundamental questions in physics especially the role of quantum fluctuations, quantum mechanics of interacting electrons, the quantum classical crossover, and the gradual buildup of macroscopic classical behavior. In these systems electron retains its quantum coherence over the entire sample and thus classicaldaws which hold in the macroscopic regime breakdown 1 2.3. 3 . Some of them being breakdown of classical Ohm's law, sample specific resistance and its fluctuations, quantization of point contact conductance, breakdown of Onsager's reciprocity relations, etc.

If the system dimensions are less than the electron phase relaxation length then electron retains its phase coherence and scattering in the sample is only elastic, indeed electronic motion in such systems is not unlike light propagation in waveguide structures and Fabry-Perot interferometers. The study of open and closed mesoscopic rings has given rise to some surprising findings. In closed mesoscopic rings pierced by a magnetic flux persistent currents have been predicted 5 and also experimentally seen 6 . The magnetic flux destroys the time-reversal symmetry and as a consequence persistent current flows in a ring. These currents are periodic in magnetic flux, with a period $\Phi_{0}, \Phi_{0}$ being the elementary flux quanta $\left(\Phi_{0}=\frac{h c}{e}\right)$. At zero temperature in a ballistic ring of circumference $l$ the amplitude of persistent current is given by $\frac{e v_{f}}{l}$, where $v_{f}$ is the Fermi velocity. For, spinless electrons, the persistent current can be either diamagnetic or paramagnetic depending upon whether the total number of electrons present in the isolated ring is odd or even respectively. This behavior of the persistent current is also known as the parity effect. In open rings pierced by the same magnetic flux, normal state Aharonov-Bohm (AB) oscillations have been observed apart from this currently these $\mathrm{AB}$ oscillations in open rings are at the heart of a large number of experiments, especially some wherein the quantum phase shift is purported to have been measured by inserting a quantum dot in one of the arms of the ring 9 and measurement of which path detection which is crucial in understanding the nature of dephasing via the role of quantum entanglement.

Another, purely quantum mechanical phenomena in such mesoscopic rings although in the absence of Amex is that of current enhancement or magnification 1011.12. Current enhancement can be defined as follows- In a metallic loop connected to two reservoirs at chemical potentials $\mu_{1}$ and $\mu_{2}$ (with $\mu_{1}>\mu_{2}$ ) by means of two ideal leads (as in inset of figure 1), transport current $I$ flows through the system. This transport current divides into $I_{1}$ and $I_{2}$ in the upper and lower arms of the ring. In classical case both $I_{1}$ and $I_{2}$ are positive and flow in the same direction as the input transport current. In quantum mechanics, however for particular values of Fermi energy intervals $I_{1}$ or $I_{2}$ can become much larger than I, this implies to obey Kirchoff's law the current in the other loop must be negative. This property that current in one of the arms is larger than the transport current is referred to as current enhancement effect. In this situation, we interpret the negative current flowing in one arm of the ring as a circulating current that flows continually in the loop. When the negative current flows in the upper arm the circulating current direction is taken to be anti-clockwise (or negative) and when it flows in the lower arm the circulating current direction is taken to be clockwise (or positive) 13. Studies on current magnification effect in mesoscopic open rings have been extended to thermal current 14 and fo spin currents in the presence of Aharonov-Casher flux 15. Recently this effect has been studied in presence of spin-flipgattering which causes dephasing of electronic motion 1210.

The current enhancement effect leads to an enhanced magnetic response (orbital magnetic moment) of a loop carrying current in the absence of magnetic flux日. It is to be noted that these circulating currents arise in the absence of magnetic flux, however, in presence of transport currents (i.e., in a non-equilibrium system). In the present work our thrust is whether we can observe the 
aforesaid current enhancement effect and the resulting circulating currents in equilibrium. For this we consider the system as depicted in figure 1. The static localised flux piercing the loop is necessary to break the time reversal symmetry and induce a persistent current in the system. The geometry we consider is a one-dimensional ring with an attached bubble and a lead connected to a reservoir at chemical potential $\mu$, for simplicity we have ignored the electron-electron interaction. The reservoir acts as an inelastic scatterer and as a source of energy dissipation. All the scattering processes in the leads including the loop are assumed to be elastic. Hence there is a complete spatial separation between the elastic and inelastic processes. The loops J1J2aJ3J1 and J1J2bJ3J1 enclose the localised flux $\Phi$. However, the bubble J2aJ3bJ2 does not enclose the flux $\Phi$. We have considered this special topology in order to verify the existence of circulating currents at equilibrium. We show that circulating currents (due to current enhancement) arise in a bubble which does not enclose a magnetic flux. We would like to mention here that the current enhancement effect and the associated circulating currents arise even when the magnetic field extends over the entire sample. However, for this the treatment is involved as one has to study separately persistent as well as circulating currents in the bubble as they have different symmetry properties. This has been studied in a simple loop in the presence of both transport currents and magnetic flux 13 . Just for the sake of simplicity and to show the existence of current enhancement in equilibrium we have taken a system in which bubble does not enclose a magnetic flux, which may not be an ideal system. However, it clarifies our contention.

In the local coordinate system the wave-functions in the various regions of our system are given as follows 17 .

$$
\begin{aligned}
& \psi_{0}=e^{i k x_{0}}+r e^{-i k x_{0}} \\
& \psi_{j}=a_{j} e^{i\left(k+\frac{\alpha_{j}}{l_{j}}\right) x_{j}}+b_{j} e^{-i k x_{j}+i \frac{\alpha_{j}}{l_{j}}\left(x_{j}-l_{j}\right)} .
\end{aligned}
$$

Here $x_{j}, j=0,1 . .4$ are coordinates along the connecting lead to the reservoir, the segments J1J2, J2aJ3, J2bJ3, and J3J1, while $\alpha_{j}$ 's are the phases picked up by the electron as it traverses the various regions of the system and $l_{j}$ 's are the lengths of the segments J1J2, J2aJ3, J2bJ3, and J3J1 respectively. The wave-vector is defined as $k=\sqrt{2 m E / \hbar^{2}}$. To solve for the unknown coefficients in eqn. (1) we use Griffith 18,1920 boundary condition at the junctions $J 1, J 2$ and $J 3$. These boundary conditions (given in eqn. 2 below) are due to the single-valuedness of wavefunction and conservation of current (Kirchoff's law).

$$
\begin{gathered}
\psi_{0}\left(x_{0}=0\right)=\psi_{1}\left(x_{1}=0\right)=\psi_{4}\left(x_{4}=l_{4}\right), \\
\psi_{0}^{\prime}\left(x_{0}=0\right)+\psi_{4}^{\prime}\left(x_{4}=l_{4}\right)=\psi_{1}^{\prime}\left(x_{1}=0\right), \\
\psi_{1}\left(x_{1}=l_{1}\right)=\psi_{2}\left(x_{2}=0\right)=\psi_{3}\left(x_{3}=0\right),
\end{gathered}
$$

$\mathrm{h}$

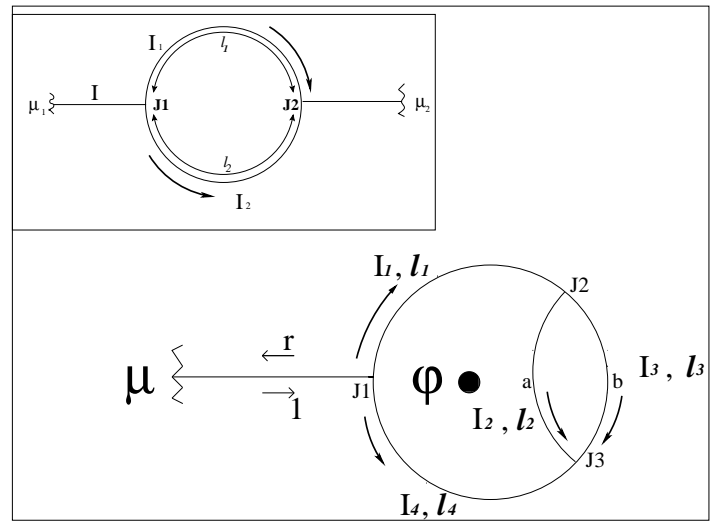

FIG. 1: The hybrid ring system connected to a reservoir at chemical potential $\mu$. The bubble is denoted by the structure J2bJ3aJ2.The localised flux $\Phi$ penetrates the ring. The current densities in various parts of the structure are denoted by I's while the lengths of the various regions are denoted by l's. In the inset we have shown the non-equilibrium case, a one dimensional mesoscopic ring with leads is connected to two reservoirs at chemical potentials $\mu_{1}$ and $\mu_{2}$.

$$
\begin{gathered}
\psi_{1}^{\prime}\left(x_{1}=l_{1}\right)=\psi_{2}^{\prime}\left(x_{2}=0\right)+\psi_{3}^{\prime}\left(x_{3}=0\right), \\
\psi_{2}\left(x_{2}=l_{2}\right)=\psi_{3}\left(x_{3}=l_{3}\right)=\psi_{4}\left(x_{4}=0\right), \\
\psi_{2}^{\prime}\left(x_{2}=l_{2}\right)+\psi_{3}^{\prime}\left(x_{3}=l_{3}\right)=\psi_{4}^{\prime}\left(x_{4}=0\right) .
\end{gathered}
$$

Herein $\psi_{j}^{\prime}\left(x_{j}=l_{j}\right)$ denotes $\left[\left(\frac{\partial}{\partial x_{j}}-\frac{i \alpha_{j}}{l_{j}}\right) \psi_{j}\right]_{x_{j}=l_{j}}$. Using the above mentioned boundary conditions we get-

$$
\begin{array}{r}
1+r=a_{1}+b_{1} e^{-i \alpha_{1}}=a_{4} e^{i k l_{4}+i \alpha_{4}}+b_{4} e^{-i k l_{4}} \\
1-r-a_{1}+b_{1} e^{-i \alpha_{1}}+a_{4} e^{i k l_{4}+i \alpha_{4}}-b_{4} e^{-i k l_{4}}=0 \\
a_{1} e^{i k l_{1}+i \alpha_{1}}+b_{1} e^{i k l_{1}}=a_{2}+b_{2} e^{i \alpha_{2}}=a_{3}+b_{3} e^{i \alpha 3} \\
a_{1} e^{i k l_{1}+i \alpha_{1}}-b_{1} e^{-i k l_{1}}-a_{2}+b_{2} e^{-i \alpha_{2}}-a_{3}+b_{3} e^{-i \alpha_{3}}=0 \\
a_{2} e^{i k l_{2}+i \alpha_{2}}+b_{2} e^{-i k l_{2}}=a_{3} e^{i k l_{3}+i \alpha_{3}}+b_{3} e^{-i k l_{3}}=a_{4}+b_{4} e^{-i \alpha_{4}} \\
a_{2} e^{i k l_{2}+i \alpha_{2}}-b_{2} e^{-i k l_{2}}+a_{3} e^{i k l_{3}+i \alpha_{3}}-b_{3} e^{-i k l_{3}}-a_{4}+b_{4} e^{-i \alpha_{4}}=0 .(3)
\end{array}
$$

Here $\alpha_{1}, \alpha_{2}, \alpha_{3}$ and $\alpha_{4}$ are phases picked up by the wave-functions in the segments J1J2, J2aJ3, J2bJ3 and J3J1 respectively and we have $\alpha_{1}+\alpha_{2}+\alpha_{4}=2 \pi \Phi / \Phi_{0}$, and $\alpha_{1}+\alpha_{3}+\alpha_{4}=2 \pi \Phi / \Phi_{0}$ such that $\alpha_{2}=\alpha_{3}$ as required by definition. Using eqn. (3) we have solved for all the unknown coefficients in eqn. (1).

In the lead connecting the reservoir to our circuit there is no current flow as $|r|^{2}=1$. The current densities $\left(I_{j}\right.$, in a dimensionless form) 21 in the small interval $d k$ around the Fermi energy $k$ in the various segments of the circuit are given by- $I_{j}=\left|a_{j}\right|^{2}-\left|b_{j}\right|^{2}$. The current densities are calculated from the usual formula of current density in presence of magnetic flux- 


$$
J_{j}=\frac{e \hbar}{2 m i}\left(\psi_{j}^{*} \nabla \psi_{j}-\psi_{j} \nabla \psi_{j}^{*}-\frac{2 i \alpha_{j}}{l_{j}} \psi_{j}^{*} \psi_{j}\right),
$$

which implies $I_{j}=\frac{J_{j}}{e \hbar k / m}$.

The persistent current densities in various parts of the circuit show cyclic variation with flux and $\Phi_{0}$ periodicity, and oscillate between positive and negative values as a function of energy or the wavevector $k$ as expected. Since the analytical expressions for these currents are too lengthy we confine ourselves to a graphical interpretation of the results. It should be noted that in all these expressions for current densities flux enters only through the combinations $\alpha_{1}+\alpha_{2}+\alpha_{4}$ and $\alpha_{1}+\alpha_{3}+\alpha_{4}$ the magnitude of these combinations is given by $2 \pi \Phi / \Phi_{0}$ as expected. For us the current densities in the bubble, i.e., J2bJ3a.J2 are of special importance as in this region there is a possibility of current enhancement which will be analysed below. The currents flowing in segment J3J1 and J1J2 are equal, i.e., $I_{1}=I_{4}$. These currents may have positive (clockwise) or negative (anti-clockwise) values depending on the flux $\Phi$ and value of wavevector $k$. For a fixed $k$ this current oscillates between positive and negative values as a function of $\Phi$ with a period $\Phi_{0}$ and are asymmetric in $\Phi$. Similarly for fixed value of $\Phi$ currents oscillate as one varies $k$. The magnitude of current shows a maximum or minimum near the corresponding eigen-states of the system. We have calculated these eigen states for two different cases. For open system as depicted in figure 1 one can calculate the energies (or wave-vector) of these states by looking at the complex poles of the S-Matrix. In our case S-Matrix is simply a complex reflection amplitude $r$. We have also analysed the eigen states of a closed system (without coupling lead to reservoir) by wave-function matching in various segments using waveguide theory.

We analyse the case of a bubble with unequal lengths, of its two arms, i.e., the length of $J 2 b J 3 \neq J 2 a J 3$. This asymmetry implies that currents in the two arms of the bubble are unequal, i.e., $I_{2} \neq I_{3}$. This asymmetry is very much essential for current enhancement. In figure 2 we plot the persistent current densities in various parts of the circuit, for this we have chosen a small interval of dimensionless wavevector $k l$ and other physical parameters are indicated in the figure captions. It should be noted that the absolute value of the persistent current densities $I_{2}$ and $I_{3}$ in this interval are individually much larger than the input current density $I_{1}$ into the bubble and thus the current enhancement effect is evident (without violating the basic Kirchoff's law). The input current arises due to the presence of flux $\Phi$ as it breaks the time reversal symmetry. In the interval $5.2<k L<7.4$ current $I_{1}$ changes from positive to negative and exhibits extremum around the real part of the poles of the S-Matrix. When $I_{1}$ is positive, negative current density of magnitude $I_{2}$ flows in the arm $J 2 a J 3$ of the bubble. Thus, when $I_{1}$ is positive circulating current flows in the clockwise direction in the bubble. In the range where $I_{1}$ is negative, i.e., input current into the bubble is in an anti-clockwise direction,

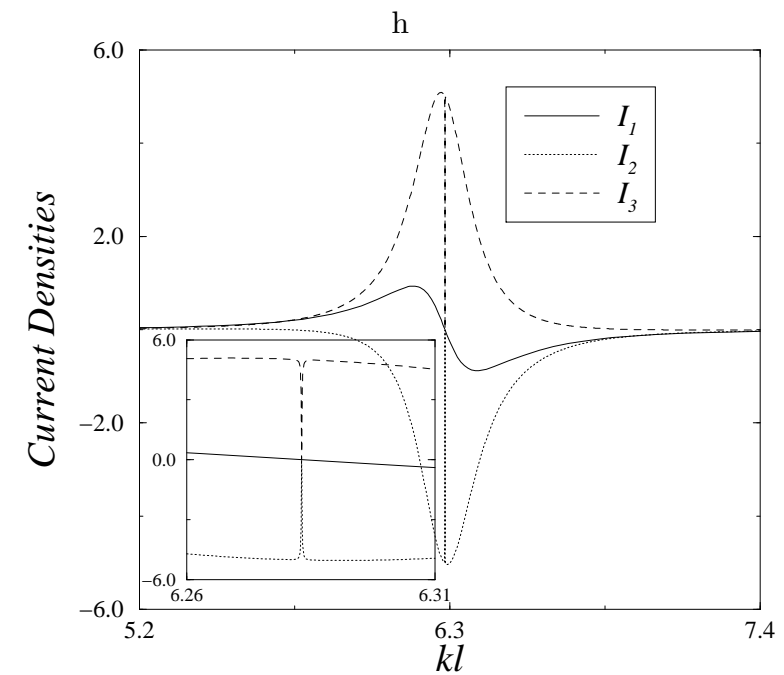

FIG. 2: Current enhancement shown with lengths $l_{1} / l=$ $l_{4} / l=0.75, l_{2} / l=0.45, l_{3} / l=0.55$. Herein the persistent current densities in the various parts of the circuit are plotted. The persistent current density in $J 1 J 2$ is denoted by the solid line while those in $J 2 a J 3$ and $J 2 b J 3$ are denoted by dotted and dashed line. Flux $\alpha=2 \pi \Phi / \Phi_{0}=0.1$.

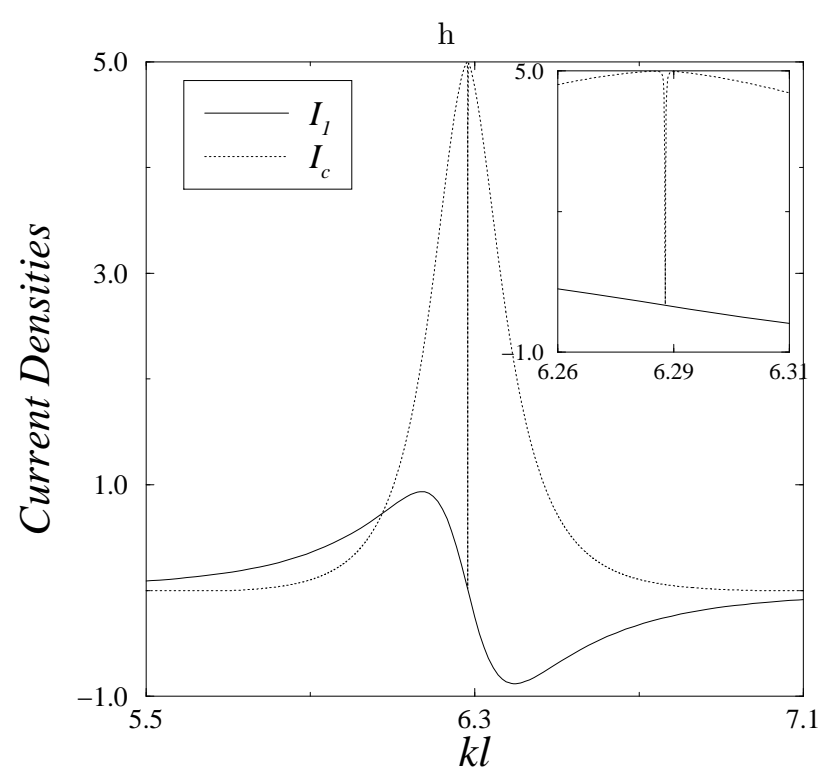

FIG. 3: Persistent current density $I_{1}$ and circulating current density $I_{c}$ is plotted as function of $k l$. The parameters are same as used in fig. 2. The inset shows the behavior of $I_{c}$ and $I_{1}$ around their zero values.

then positive current flows in arm J2bJ3. According, to our convention as mentioned earlier, circulating current flows in the clockwise direction. The magnitude of this circulating current $I_{c}$ (negative current), is taken to be the value of current in one of the arms of the bubble moving against the input current into the bubble as explained in detail in the introduction. In all the figures drawn the 


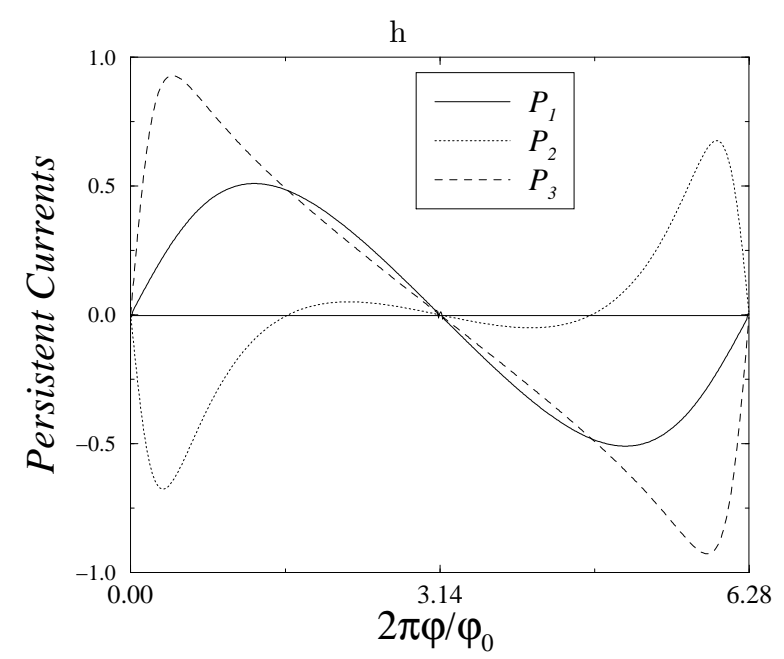

FIG. 4: Current enhancement shown with lengths $l_{1} / l=$ $l_{4} / l=0.25, l_{2} / l=0.45, l_{3} / l=0.55$. Herein the persistent currents in the various parts of the circuit are plotted as function of flux. The Fermi wavevector here is $k_{f}=4 \pi$.

length of the bubble $l=l_{2}+l_{3}$ is taken as unity, and the current densities along with the Fermi wave-vectors are in their dimensionless form. This current enhancement effect is extremely sensitive to the lengths of the arms of the bubble. In figure 3 we have plotted the persistent current density $I_{1}=I_{4}$ and the circulating current density $I_{c}$ in the bubble for the same parameters used in figure 2. For figure 3 we have used a smaller scale of $k l$ for clarity. Even though persistent current density $I_{1}$ changes the sign as we cross the real part of the pole the circulating current remains clockwise only. Moreover, $I_{c}$ around either side of the poles has a magnitude larger than the absolute magnitude of $I_{1}$. It should be noted that if we interchange the values of $l_{2}$ and $l_{3}$ keeping other parameters unchanged circulating current will flow in an anti-clockwise direction. This is obvious from the geometry of the problem. Alongwith the current densities the total persistent currents in various parts of the ring can also be plotted, to do that we integrate the current densities $J_{j}$ in various regions of the circuit over the Fermi wave vector. The persistent currents $P_{j}$ at temperature $T=0$ is given by

$$
P_{j}=-\int_{0}^{k_{f}} J_{j} d k
$$

In figure 4 we have plotted the persistent currents (in dimensionless units) as a function of flux for a fixed value of $k_{f}$ and the system parameters are indicated in the figure caption as expected persistent currents in various arms are flux periodic and asymmetric in flux. Here also we can see the persistent current enhancement effect in some range of flux $\Phi$.

We generally observe enhanced currents at those Fermi energy wave-vector intervals which are around the poles

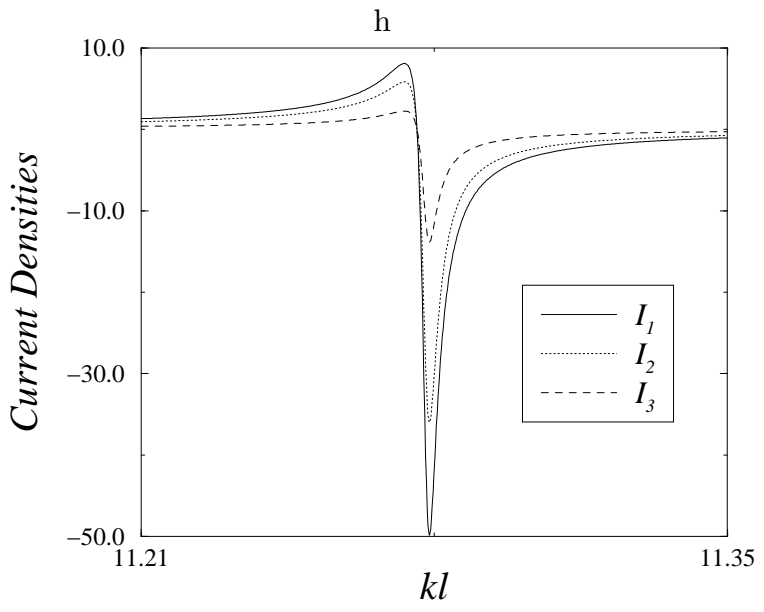

FIG. 5: Absence of current enhancement shown with lengths $l_{1} / l=l_{4} / l=0.75, l_{2} / l=0.25, l_{3} / l=0.75$. Herein the persistent current densities in the various parts of the circuit are plotted. The persistent current density in $J 1 J 2$ is denoted by the solid line while those in $J 2 a J 3$ and $J 2 b J 3$ are denoted by dotted and dashed line. The $k l$ value 11.28 is an eigen wavevector of the closed system. Flux $=0.1$.

of the open system 19, 113 . However, there are some exceptions. In figures 5 and 6 we plot a few of those exceptions. We consider the lengths $l_{1} / l=l_{4} / l=0.75, l_{2} / l=$ 0.35 and $l_{3} / l=0.65$ and flux $\alpha=\frac{2 \pi \Phi}{\Phi_{0}}=0.1$. In figures 5 and 6 we show that current enhancement effect does not occur at places which are eigen values of the aforesaid system. Here the eigen wave-vector $k L$ corresponds to 11.28 in figure 5 and 13.85 in figure 6 . One can readily notice that the magnitude of persistent current density (i.e., input current density $I_{1}$ ) shows extrema around this value. Around this region the current densities in the bubble $I_{2}$ and $I_{3}$ are individually smaller than $I_{1}$ and they flow in the same direction as the input current. Hence we do not observe current enhancement effect around the quasi bound states for these parameters of the open system. The exact conditions for current enhancement cannot of course be readily predicted a priori. If system exhibits current enhancement one should be able to detect it experimentally by observing the enhanced response of the magnetic moment.

As mentioned in the paragraphs above, current enhancement directly corresponds to resonances of our system. These resonances occur at or near the quasi bound states of the open system. Thus it is important to study the closed system analog to see where these bound states occur and how are they linked to the quasi bound states. More than that the study of bound states of the closed system will give us the eigen energy spectra from which many important parameters like magnetic moment and persistent currents can be evaluated. In figures 7,8 and 9 we have plotted the first few eigen energies $E=k_{n}^{2}$ of our isolated system (with the connecting lead to reservoir removed). The parameters are mentioned in the figure 


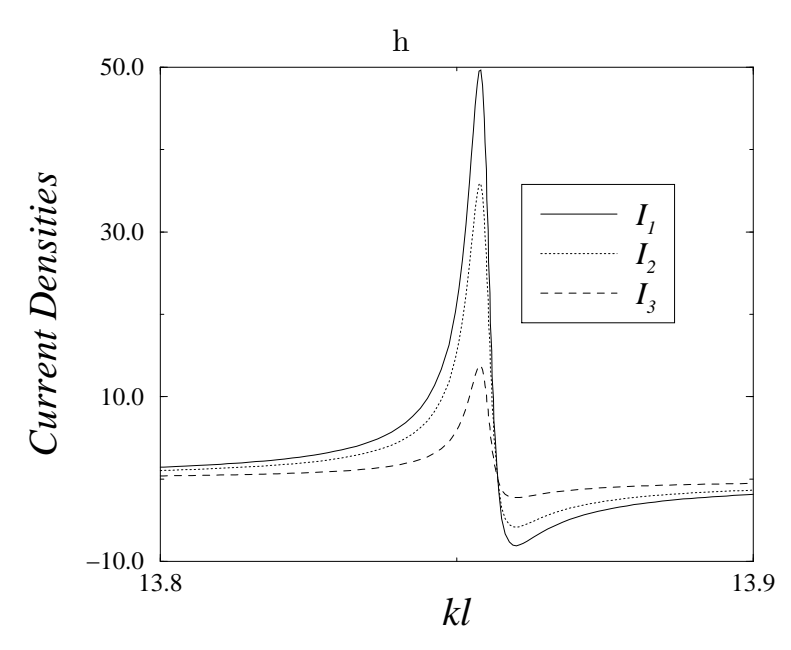

FIG. 6: Absence of current enhancement shown with lengths $l_{1} / l=l_{4} / l=0.75, l_{2} / l=0.25, l_{3} / l=0.75$. Herein the persistent current densities in the various parts of the circuit are plotted. The persistent current density in $J 1 J 2$ is denoted by the solid line while those in $J 2 a J 3$ and $J 2 b J 3$ are denoted by dotted and dashed line. The $k l$ value 13.85 is an eigen wavevector of the closed system. Flux $=0.1$.

captions. These eigen energies are calculated with the help of quantum waveguide approach from the condition that the determinant of the coefficient matrix must vanish. The coefficient matrix is built from first principles using quantum waveguide theory with the second wavefunction of equation 1 . The condition for bound states of the system, i.e., the closed ring with bubble is given by

$$
\begin{aligned}
\cos (\alpha)= & \frac{1}{\cos \left(k l_{-}\right)}\left(\cos k\left(l_{1}+l_{+}\right)-\right. \\
& \left.\frac{1}{4} \frac{\sin \left(k l_{1}\right) \sin \left(k l_{2}\right) \sin \left(k l_{3}\right)}{\sin \left(k l_{+}\right)}\right)
\end{aligned}
$$

where $\alpha=2 \pi \Phi / \Phi_{0}, l_{+}=\left(l_{2}+l_{3}\right) / 2$ and $l_{-}=$ $\left(l_{2}-l_{3}\right) / 2$. The eigen energies are flux periodic. The persistent current carried by a electron in the eigen state $E_{n}$ is given by $I_{n}=-\frac{1}{c} \frac{\partial E_{n}}{\partial \Phi}$. In a closed single loop persistent current changes its sign as we go from one level to the next successive level. Thus for spinless electrons the persistent current is diamagnetic or paramagnetic depending on total number electrons being odd or even(at $T=0)$. This is called parity effect. Supposing we open up the ring and find its transmission amplitude $t$ then we can easily see that

$$
\cos (\alpha)=\operatorname{Re}(1 / t)
$$

In our system with the help of eqn. 7 we can calculate the persistent current carried by an electron in the eigen state $E_{n}$. This is given by- $I_{n}=-\frac{e}{\hbar} \frac{\sin (\alpha)}{d \operatorname{Re}(1 / t) / d E_{n}}$. From $\mathrm{h}$

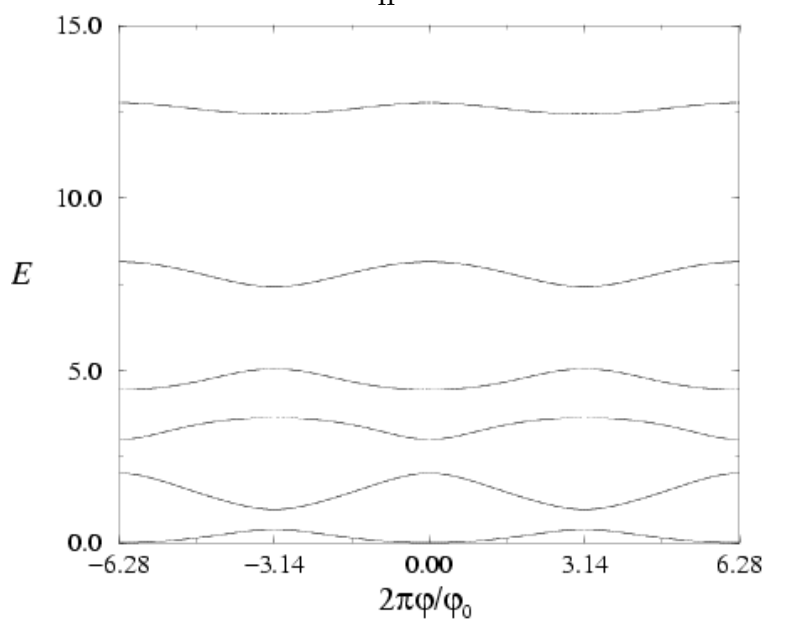

FIG. 7: Breakdown of parity effects in a closed one dimensional mesoscopic ring coupled to a bubble. The lengths are $l_{1} / l=0.75, l_{2} / l=0.35, l_{3} / l=0.65$. The energies are normalised by $\pi^{2}$.

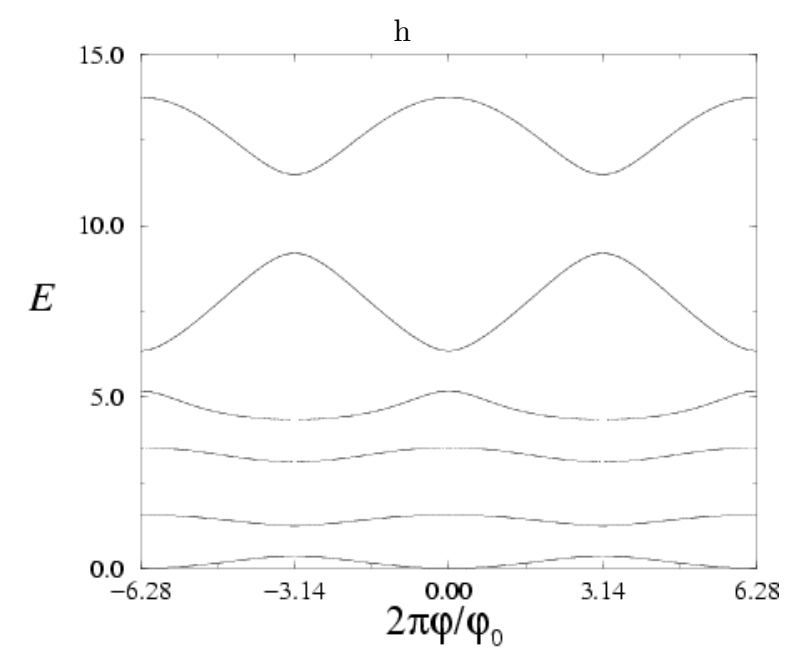

FIG. 8: Breakdown of parity effects in a closed one dimensional mesoscopic ring coupled to a bubble. The lengths are $l_{1} / l=0.75, l_{2} / l=0.15, l_{3} / l=0.85$. The energies are normalised by $\pi^{2}$.

the above formula we get for the persistent currents in our closed system the following expression-

$$
\frac{\hbar I_{n}}{e}=\frac{-8 k_{n} \sin (\alpha) \cos ^{2}\left(k_{n} l_{-}\right) \sin ^{2}\left(k_{n} l_{+}\right)}{D}
$$

where $D=\sin \left(k_{n} l_{3+}\left(16 l_{2+}+2 l_{3+}\right)+\sin \left(k_{n} l_{2+}\left(16 l_{3+}+\right.\right.\right.$ $\left.2 l_{2+}\right)+\sin \left(k_{n} l_{3-}\right)\left(-6 l_{3}-10 l_{1}+2 l_{3-} \cos \left(2 k_{n} l_{2}\right)\right)+$ $\sin \left(k_{n} l_{2-}\right)\left(-6 l_{2}-10 l_{1}+2 l_{2-} \cos \left(2 k_{n} l_{3}\right)\right) \quad-$ $l_{3+}\left(\sin \left(k_{n} l_{3+}-2 k_{n} l_{2}\right)+9 \sin \left(k_{n} l_{3+}+2 k_{n} l_{2}\right)-\right.$ $l_{2+}\left(\sin \left(k_{n} l_{2+}-2 k_{n} l_{3}\right)+9 \sin \left(k_{n} l_{2+}+2 k_{n} l_{3}\right)\right)$ and $l_{i \pm}=\left(l_{1} \pm l_{i}\right)$, herein $i=2,3$ and $k_{n}$ are the eigen wave vectors. 


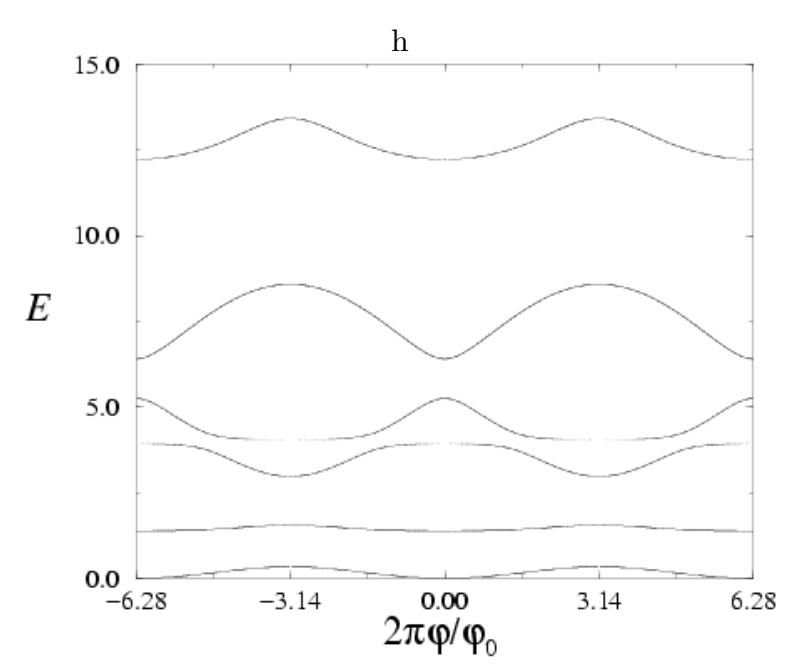

FIG. 9: Breakdown of parity effects in a closed one dimensional mesoscopic ring coupled to a bubble. The lengths are $l_{1} / l=0.75, l_{2} / l=0.05, l_{3} / l=0.95$. The energies are normalised by $\pi^{2}$.

With the help of $k_{n}$ one can easily evaluate the persistent currents from eqn. 8 for some representative values as in figures 7,8 or 9 . The eigen energies are flux periodic with period $\Phi_{0}$. It can be noted from figure 7 that 3rd and 4th eigen states carry diamagnetic current while 5th and 6th carry paramagnetic current for small values of flux (which is obvious from their slopes). Thus breaking the well known parity effect. For details we refer to Ref. 22]. Similarly, in figures 8 and 9 this violation of parity effects is also seen but of course the eigen spectra is modified, although the length of the bubble $l=l_{2}+l_{3}=1$ still holds as well as the fact that the length of the outer arm is also same as in figure 7 , what have been altered are the arm lengths of the bubble, within the restriction that the length of the bubble is unity. This goes on to show that the eigen energies are sensitive to the arm lengths of the bubble. We have later on tabulated some of the eigen $k$ vectors for all these three cases as in the three figures.

A comparison of the eigenenergy spectra and the persistent currents in the open system is herein called for so as to assess for ourselves whether the persistent currents (global) calculated from the eigenenergy spectra and inherently linked to the slope of the energy versus flux diagram are same as the the persistent currents (local) in the various arms of the system calculated from the eqn. (3). Figures 10, 11 and 12 depict the persistent current densities in the open system for the same parameters as in figures 7, 8 and 9 respectively. These persistent current densities are continuous functions of the dimensionless wavevector $k l$. We have chosen a smaller scale for the magnitude of the current densities for better visibility of the detailed features. An important conclusion which can be drawn from the comparisons is that resonances, in the persistent current densities versus energy graph, arise at

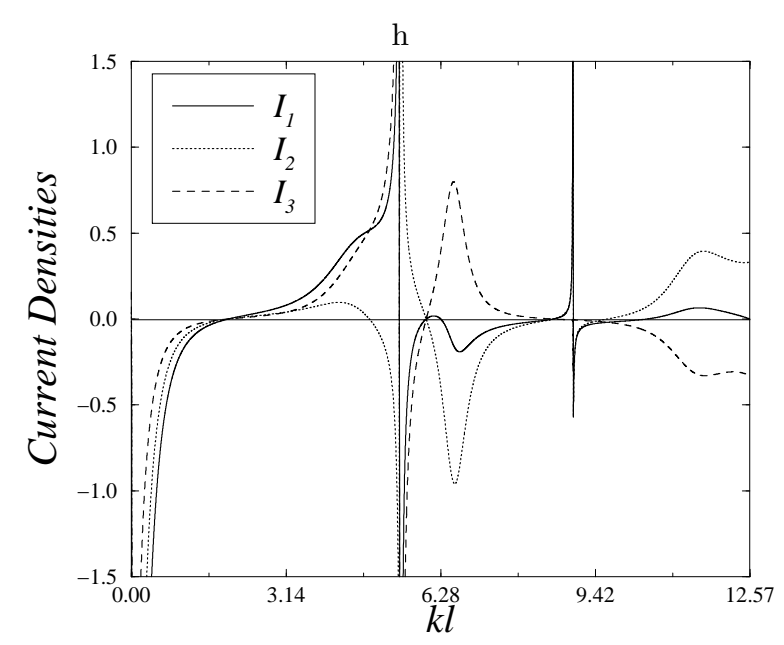

FIG. 10: Current densities in the open system for length parameters as in figure 7 . Flux $=0.1$.

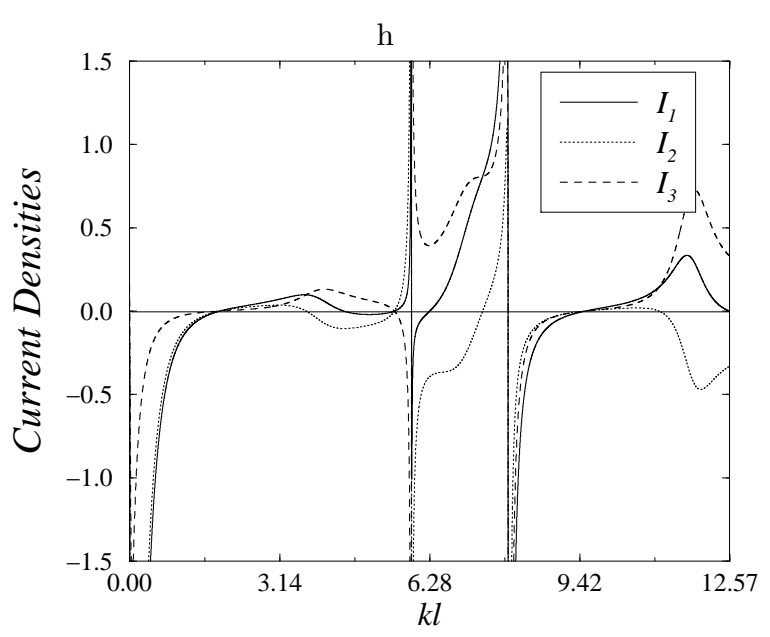

FIG. 11: Current densities in the open system for length parameters as in figure 8 . Flux $=0.1$.

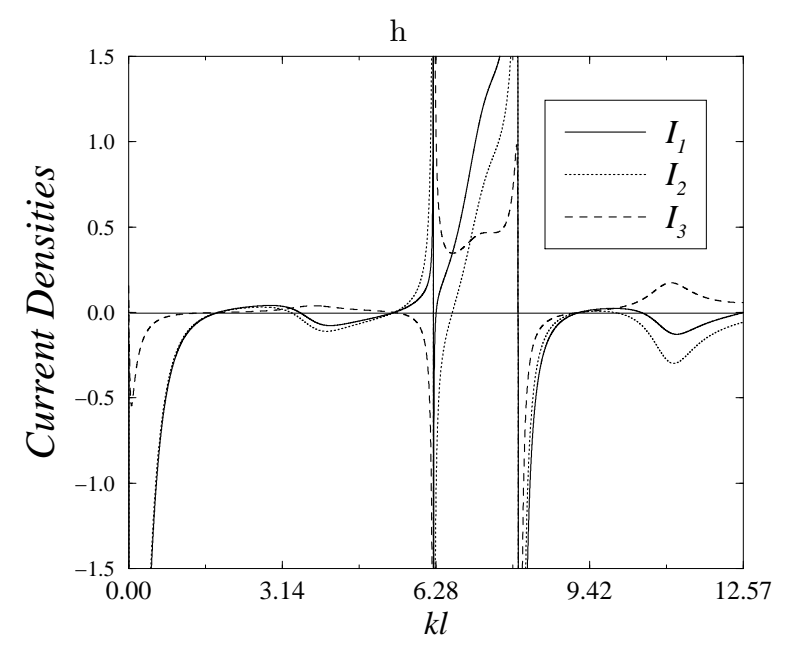

FIG. 12: Current densities in the open system for length parameters as in figure 9 . Flux $=0.1$. 


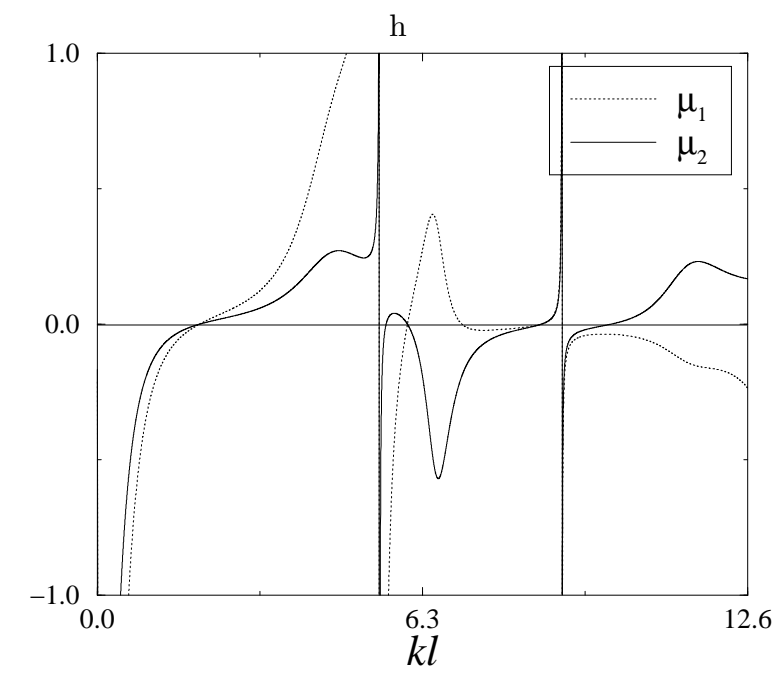

FIG. 13: Plot of $\mu_{1}$ and $\mu_{2}=I_{g} A_{r} / 2$ for length parameters as in figures 7 and 10. Flux $=0.1$.

the bound states of the closed system of course they are shifted a bit as a result of coupling to an external reservoir. The shifted $k$ values are also later on tabulated. Another important fact to be noted is that the energies wherein current enhancement occurs at these, the local current densities can be either diamagnetic or paramagnetic and therefore are not same as the global persistent currents but at those energies wherein current enhancement is not observed therein it is observed that there is no discrepancy between local and global currents, i.e., if the eigenenergy spectra reveals diamagnetic currents the local currents are also seen to be diamagnetic. For example if we compare figures 7 and 10 we note that the closed system eigen $k$ 's are at $0.1526,4.4535,5.4574,6.627,8.912$, and 11.234. Around these eigen $k$ 's we see from figure 10 that those $k$ values where current enhancement does not take place therein there is no difference between the nature (being diamagnetic or paramagnetic) of global and local current densities in different segments but wherein current enhancement occurs therein there is a difference between the nature of global and local current densities (by definition current enhancement implies this, as the current densities in the two arms of the bubble are in opposite direction).

Extending our discussion to the magnetic moment densities of our system we see that the magnetic moment densities calculated for the open system do not seem to agree at least qualitatively with that calculated from the eigen energy spectra in some energy interval. Furthermore the orbital magnetic moment density defined via currents in a loop, depends strongly on the topology of the system, whereas eigen spectrum do not. In fact there are infinitely many topological structures possible. If we consider our system as depicted in figure 1 to be planar and lying in the $\mathrm{x}-\mathrm{y}$ plane then the magnetic moment density $\left(\mu_{1}\right)$ can be viewed as being generated by current

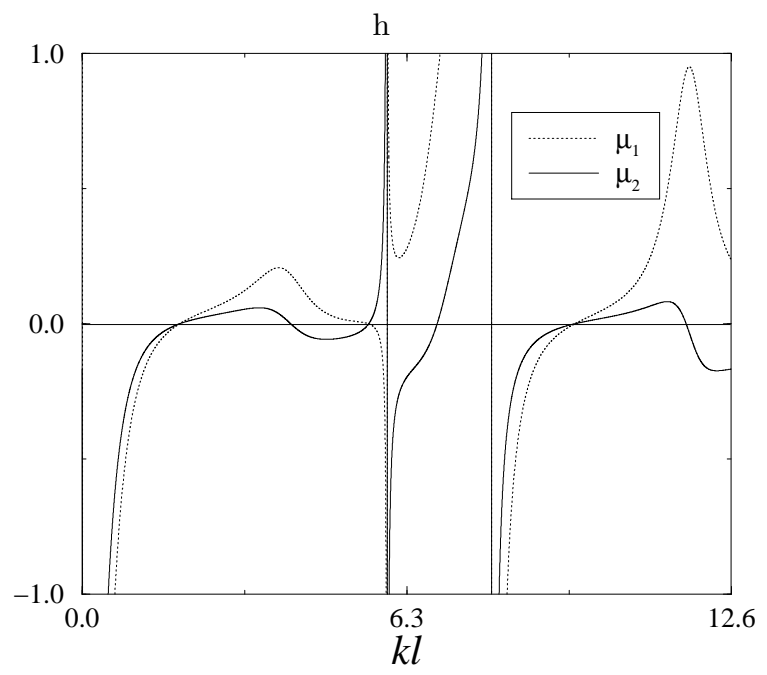

FIG. 14: Plot of $\mu_{1}$ and $\mu_{2}=I_{g} A_{r} / 2$ for length parameters as in figures 8 and 11. Flux $=0.1$.

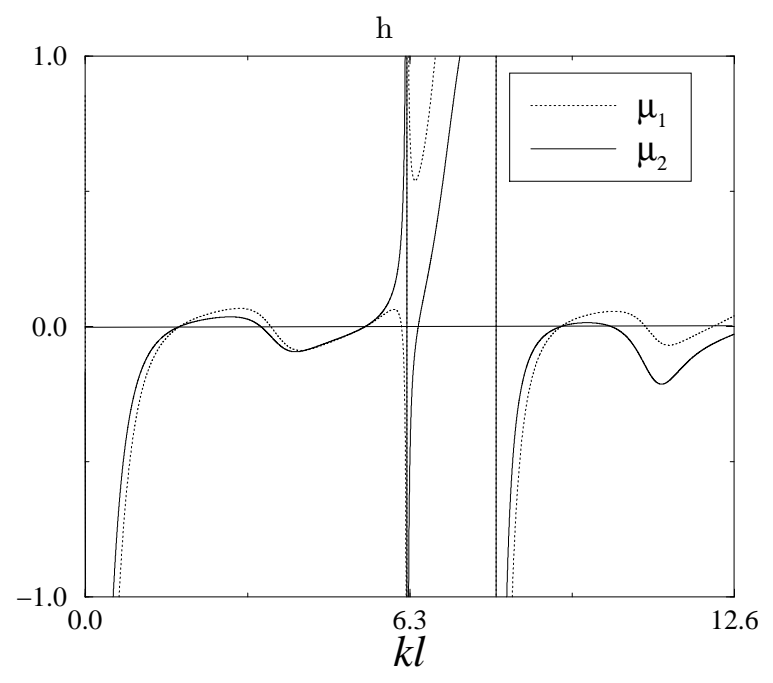

FIG. 15: Plot of $\mu_{1}$ and $\mu_{2}=I_{g} A_{r} / 2$ for length parameters as in figures 9 and 12. Flux $=0.1$.

density $I_{1}$ enclosing an area $A_{r}$ and by current density $I_{3}$ enclosing area $A_{b}$, i.e., $\mu_{1}=\frac{1}{c}\left(I_{1} A_{r}+I_{3} A_{b}\right)$, wherein $A_{r}$ and $A_{b}$ are areas enclosed by the ring $(J 1 J 2 a J 3 J 1)$ and bubble(J2aJ3bJ2) respectively. Another orientation of the system in which the arm $J 2 b J 3$ is in the $\mathrm{x}-\mathrm{z}$ plane gives $\mu_{2}=\frac{1}{c}\left(I_{1} A_{r}+I_{2} A_{r}\right) / 2=I_{g} A_{r} / 2$ wherein $I_{g}=I_{1}+I_{2}$ is said to be the most appropriate generalization of the equilibrium persistent current, see 23 for further details, and this along with $\mu_{1}$ is what we plot in figures 13, 14 and 15. In these figures, the plot of $\mu_{1}$ has been magnified a hundred times for better visibility. The physical parameters in these figures, i.e., 13, 14 and 15 are same as that in figures 7,8 and 9 respectively (also figures 10, 11 and 12 respectively). Of course $I_{g}$ is proportional to $\mu_{2}$. Several other orientations also are 
possible, for example, if the bubble lies in x-y plane and the ring lies in X-z plane, then $\mu_{z}=\frac{1}{c}\left(I_{3} A_{r}-I_{2} A_{r}\right) / 2$ and $\mu_{y}=\frac{1}{c} I_{1} A_{r}$. Even when our system lies in the x-y plane for fixed $l_{1}, l_{2}, l_{3}$ and by deforming their shapes we can have different values of magnetic moment density along the $\mathrm{z}$ direction. The energy eigen values remain intact inspite of the deformation. All the above examples buttress the fact that the orbital magnetic moment is inherently linked to the topology of the system. As we have earlier pointed out the magnetic moment calculated herein is not same (qualitatively), as the magnetic moment calculated in case of the closed system from its eigenenergy spectra, which is same for all topological situations. Some important conclusions can be drawn from the figures 13, 14 and 15 , first $\mu_{1}$ and $\mu_{2}$ are not same and also one can notice that in some $k l$ intervals $\mu_{1}$ and $\mu_{2}$ have opposite signs, i.e., the nature of magnetic moment is qualitatively different for these two cases. Thus this leads to a contradiction as to with which of these can one associate the magnetic moment. The magnetic moment is fixed for closed system from the energy eigenvalue spectrum calculated from $\mu=-\frac{1}{c} \frac{\partial E_{n}}{\partial H}$ wherein $H$ is the magnetic field enclosed by the system. In the open system we expect behavior as a function of $k l$ to reflect the behavior in the closed system except for the fact that we get currents for all the $k l$ wave vectors. This arises from the fact that in open system life time of a particle in an eigen state is finite due to coupling to the reservoir and hence all the energy eigenvalues associated with the closed system get broadened. Again a comparison between the eigenenergy spectra, and hence magnetic moment drawn in figures 7,8 and 9 and the figures for magnetic moment densities (see, figures 13, 14 and 15) shows that in some cases the range wherein successive energy eigenvalue slopes are similar (i.e., either paramagnetic/diamagnetic) the magnetic moment densities (calculated from currents, e.g., $\mu_{1}$ or $\mu_{2}$ ) can change their nature. Some cases wherein such changes occur have been tabulated in the tables 1,2 and 3. In several cases we notice that magnetic moment densities $\mu_{1}$ or $\mu_{2}$ have different nature (directions) as compared to $\mu$ which is obtained from the slope of the energy eigenvalue spectrum. We would have expected the same for the magnetic moments calculated from the currents in the isolated system. The coupling to external reservoir does not change the nature of the currents although it broadens and shifts a bit the energy eigenvalue spectrum. Thus, the study of magnetic moment densities of the open system is enough to convey to us the picture about the magnetic moment calculated from currents in the isolated system. Hence clarifying our contention. Thus it is clear that the nature of magnetic moment densities $\mu_{1}$ and $\mu_{2}$ need not be related to eigenvalue spectrum in the same energy range or equilibrium magnetic moments at temperature $T=0$. This result we attribute solely to the current enhancement effect which is purely a quantum mechanical effect. In the ranges wherein there is no current enhancement, the nature of magnetic moment densities obtained by two different methods are qualitatively same, however they may differ in magnitude. It is also worth mentioning that the total magnetic moment (at temperature $T=0$ ) of a representative system is obtained by integrating the magnetic moment densities upto the Fermi wavevector $k_{f}$.

The preceding three paragraphs have dealt with the eigen energy spectra, persistent current densities and the magnetic moments. To have some conclusive arrivals, we juxtapose all of these in the tables 1,2 and 3. In the tables we give the detailed analysis of the eigen $k$ of the closed system, the poles of the open system, whether at these poles or bound states current enhancement takes place, what are the directions (nature) of magnetic moment densities in the open system in the small interval in the neighborhood of the quasi bound states, and the persistent currents in the closed system form calculated from eqn. 8, whose nature is similar to that of the closed system magnetic moments. In column (1) we give the value of the closed system eigen $k$, in column (2) we give the complex poles of the open system for the same lengths. The real part of the pole gives the energy at which resonance occurs while the imaginary part gives the width of the same, which of course is just the inverse of the lifetime of the particle in that resonant state. Thus smaller the imaginary part, sharper the resonance and more the particles lifetime. In column (3) we note whether enhanced currents are seen at these quasi-bound states or not. In columns (4) and (5) we mention the values of $\mu_{1}$ calculated from $I_{1} A_{r}+I_{3} A_{b}$ and $I_{g}$ in the small neighborhood of the quasi bound states. In columns for $\mu_{1}$ and $I_{g}$, para/dia implies that around that quasi bound state, nature of magnetic moment changes from being paramagnetic to the left to being diamagnetic to the right in the immediate neighborhood of this quasi bound state (in the same spirit one can understand dia/para in this column). The areas are calculated by deforming the circular arms of our system to form rectangles. Finally in column (6) we note the direction of the magnetic moment of the closed system whose nature (direction) is same as persistent currents in the closed system as calculated from eqn. 8.

\begin{tabular}{|c|c|c|c|c|c|}
\hline $\begin{array}{c}k \\
(1)\end{array}$ & $\begin{array}{c}\text { poles } \\
(2)\end{array}$ & $\begin{array}{c}\text { is current } \\
\text { enhanced }(3)\end{array}$ & $\begin{array}{c}\mu_{1} \\
(4)\end{array}$ & $\begin{array}{c}I_{g} \\
(5)\end{array}$ & $\begin{array}{c}\mu \text { from } \\
\text { eigenenergies }(6)\end{array}$ \\
\hline 0.1526 & $0.7192-0.044 i$ & no & dia & dia & dia \\
\hline 4.4535 & $4.5157-0.883 i$ & no & para & para & para \\
\hline 5.4574 & $5.4547-0.005 i$ & yes & para $/$ dia & para/dia & dia \\
\hline 6.6270 & $6.5448-0.292 i$ & yes & para & dia & dia \\
\hline 8.9728 & $8.9728-0.0004 i$ & yes & para $/$ dia & para/dia & para \\
\hline 11.234 & $11.352-0.748 i$ & yes & dia & para & para \\
\hline
\end{tabular}

TABLE I: A comparative analysis of eigen-energy spectra, current densities and magnetic moment density for $l_{1} / l=$ $l_{4} / l=0.375, l 2 / l=0.35, l 3 / l=0.65$, Refer to figures 7,10 and 13.

From the tables and graphs, one can draw some conclusions: 


\begin{tabular}{|c|c|c|c|c|c|}
\hline $\begin{array}{c}k \\
(1)\end{array}$ & $\begin{array}{c}\text { poles } \\
(2)\end{array}$ & $\begin{array}{c}\text { is current } \\
\text { enhanced }(3)\end{array}$ & $\begin{array}{c}\mu_{1} \\
(4)\end{array}$ & $\begin{array}{c}I_{g} \\
(5)\end{array}$ & $\begin{array}{c}\mu \text { from } \\
\text { eigenenergies }(6)\end{array}$ \\
\hline 0.160 & $0.162-0.571 i$ & no & dia & dia & dia \\
\hline 3.921 & $3.957-0.670 i$ & yes & para & para & para \\
\hline 5.898 & $5.898-0.001 i$ & yes & dia/para & para $/$ dia & para \\
\hline 7.136 & $7.084-0.667 i$ & no & para & para & para \\
\hline 7.948 & $7.940-0.012 i$ & no & dia & dia & dia \\
\hline 11.65 & $11.754-0.438 i$ & yes & para & para & para \\
\hline
\end{tabular}

most effective generalisation of the equilibrium persistent current is not necessarily correct. In fact we cannot think of any other topological configuration for which magnetic moments calculated from the current densities and that from the eigenvalue spectra (equilibrium magnetization) would match.

To conclude, we have shown that current enhancement effect can occur in equilibrium mesoscopic systems in

TABLE II: A comparative analysis of eigen-energy spectra, current densities and magnetic moment density for $l_{1} / l=$ $l_{4} / l=0.375, l_{2} / l=0.15, l_{3} / l=0.85$. Refer to figures 8,11 and 14 .

\begin{tabular}{|c|c|c|c|c|c|}
\hline $\begin{array}{c}k \\
(1)\end{array}$ & $\begin{array}{c}\text { poles } \\
(2)\end{array}$ & $\begin{array}{c}\text { is current } \\
\text { enhanced }(3)\end{array}$ & $\begin{array}{c}\mu_{1} \\
(4)\end{array}$ & $\begin{array}{c}I_{g} \\
(5)\end{array}$ & $\begin{array}{c}\mu \text { from } \\
\text { eigenenergies }(6)\end{array}$ \\
\hline 0.169 & $0.183-0.5660 i$ & no & dia & dia & dia \\
\hline 3.693 & $3.706-0.6380 i$ & yes & para & para & dia \\
\hline 6.228 & $6.228-0.0004 i$ & yes & dia/para & para/dia & para \\
\hline 7.170 & $7.160-0.6530 i$ & no & para & para & para \\
\hline 7.989 & $7.981-0.0154 i$ & no & dia & dia & dia \\
\hline 11.00 & $11.019-0.551 i$ & yes & dia & dia & dia \\
\hline
\end{tabular}

TABLE III: A comparative analysis of eigen-energy spectra, current densities and magnetic moment density for $l_{1} / l=$ $l_{4} / l=0.375, l_{2} / l=0.05, l_{3} / l=0.95$. Refer to figures 9,12 and 15.

(1). At eigen-energies where there is no current enhancement, the entries of column (5) are compatible with that of column (6) but at places where current enhancement occurs the entries may or may not match.

(2). Apart from the above inconsistency, we also observe from the graphs for eigenenergy spectra and magnetic moment and current densities that for parameters at which over successive energy levels, persistent currents are paramagnetic (for example, see figure (8)), the current (figure (11)) and magnetic moment densities (figure (14)) are not paramagnetic, i.e., in between energy levels they do change sign.

Hence, the parameter $I_{g}$ hitherto alluded to as the the presence of magnetic flux. Earlier, it was shown that this effect arises in a non-equilibrium state, i.e., in presence of transport current flow and in absence of magnetic field. This quantum effect is extremely sensitive to system parameters. Parity effects are shown to be violated in the isolated system. Apart from this we have analysed the global and local current densities of our system and shown that the orbital magnetic response of the system calculated from the current densities (and inherently linked to the topological configuration) is qualitatively not same as that calculated from the eigenenergy spectra. This fact is related to the current enhancement effect in these systems. To clarify our preceding contention it is imperative for us to study a system which does not exhibit current magnification effect. A suitable system to study these local and global currents and the differences which occur between them would be a system of coupled rings. Herein two different rings are coupled via an ideal lead as studied in Ref. [24]. In this system there is no question of current enhancement. However, currents (or magnetic moments) in the two rings may be of opposite sign, in this case one can also obtain the global currents (or magnetic moments) from the eigen energy spectra. It is worthwhile to check whether the sum of magnetic moments obtained from currents of two separate rings shows the same nature as obtained from the eigen energies of the entire system.

\section{Acknowledgments}

Authors acknowledge help of, and useful discussions with Sandeep K. Joshi.
* Electronic address: colin@iopb.res.in

† Electronic address: jayan@iopb.res.in

1 Y. Imry, Introduction to Mesoscopic Physics (Oxford University, New York, 1997).

2 S. Datta, Electronic transport in mesoscopic systems (Cambridge University Press, Cambridge, 1995).

3 P. S. Deo and A. M. Jayannavar, Pramana J. Phys.56, 439 (2001).

4 S. Washburn and R. A. Webb, Adv. Phys. 35, 375 .

${ }^{5}$ M. Buttiker, Y.Imry and R.Landauer, Phys. Lett. A 96, 365 (1983);

${ }^{6}$ L. P. Levy, G. Dolan, J. Dunsmuir and H. Bouchiat, Phys. Rev. Lett. 64, 2074 (1990).

7 V. Chandrasekhar, R. A. Webb, M. J. Brady, M. B.
Ketchen, W. J. Gallagher and A. Kleinsasser, Phys. Rev. Lett. 67, 3578 (1991).

8 D. Mailly, C. Chapelier and A. Benoit, Phys. Rev. Lett. 70, 2020 (1993).

9 O. Entin-Wohlman, A. Aharony, Y. Imry, Y. Levinson, A. Schiller, cond-mat/0108064. See also the following website for a wonderful talk on the phase shift measured in the Aharonov-Bohm Interferometer. http://online.itp.ucsb.edu/online/nano01/aharony/

10 A. M. Jayannavar and P. S. Deo, Phys. Rev. B49, 13685 (1994).

11 T. P. Pareek, P. S. Deo and A. M. Jayannavar, Phys. Rev. B52, 14657 (1995).

12 C. Benjamin, S. K. Joshi, D.Sahoo and A. M. Jayannavar, 
Mod. Phys. Lett. B15, 19 (2001).

13 A. M. Jayannavar, P. Singha Deo, and T. P. Pareek, Physica B212, 216 (1995).

14 M. V. Moskalets, Euro. Phys. Lett. 41, 189 (1998).

15 T. Choi, C. M. Ryu and A. M. Jayannavar, Int. J. Mod. Phys. B12, 2091 (1998) and cond-mat/9808245.

${ }^{16}$ S. K. Joshi, D. Sahoo and A. M. Jayannavar, Phys. Rev. B64, 075320 (2001).

17 C. M. Ryu, et.al., Int. J. Mod. Phys. B10, 701 (1996)

18 S. Griffith, Trans. Faraday. Soc. 49, 650(1953).
19 J-B. Xia, Phys. Rev. B45, 3593 (1992).

20 P. S. Deo and A. M. Jayannavar, Phys. Rev. B50, 11629 (1994);

21 M. Büttiker, Phys. Rev. B32, 1846(1985).

22 P. S. Deo, Phys. Rev. B51, 5441(1995);

23 P. Cedraschi and M. Büttiker, Phys. Rev. B63, 165312 (2001).

24 T. P. Pareek and A. M. Jayannavar, Phys. Rev. B54, 6376 (1996). 